\title{
Discours
}

Revue de linguistique, psycholinguistique et

informatique. A journal of linguistics, psycholinguistics

and computational linguistics

$15 \mid 2014$

Varia

Des « craintes légitimes » aux « craintes irrationnelles » : analyse argumentative de l'inscription des modalités appréciatives dans les textes du débat public sur les nanotechnologies

\section{Assimakis Tseronis}

\section{OpenEdition}

Journals

Édition électronique

URL : http://journals.openedition.org/discours/8949

DOI : $10.4000 /$ discours. 8949

ISSN : 1963-1723

Éditeur :

Laboratoire LATTICE, Presses universitaires de Caen

Référence électronique

Assimakis Tseronis, «Des « craintes légitimes » aux « craintes irrationnelles » : analyse argumentative de l'inscription des modalités appréciatives dans les textes du débat public sur les

nanotechnologies », Discours [En ligne], 15 | 2014, mis en ligne le 19 décembre 2014, consulté le 19 avril 2019. URL : http://journals.openedition.org/discours/8949 ; DOI : 10.4000/discours.8949

\section{(c)}

Discours est mis à disposition selon les termes de la licence Creative Commons Attribution - Pas d'Utilisation Commerciale - Pas de Modification 4.0 International. 

Revue de linguistique, psycholinguistique et informatique

Des « craintes légitimes » aux " craintes irrationnelles » : analyse argumentative de l'inscription des modalités appréciatives dans les textes du débat public sur les nanotechnologies

Assimakis Tseronis

Université d'Amsterdam

Assimakis Tseronis, "Des "craintes légitimes" aux "craintes irrationnelles": analyse argumentative de l'inscription des modalités appréciatives dans les textes du débat public sur les nanotechnologies», Discours [En ligne], 15 | 2014, mis en ligne le 19 décembre 2014. 



\section{Des « craintes légitimes » aux " craintes irrationnelles » : analyse argumentative de l'inscription des modalités appréciatives dans les textes du débat public sur les nanotechnologies}

Assimakis Tseronis

Université d'Amsterdam

Dans cet article, on étudie comment les modalités appréciatives peuvent être interprétées quand elles sont inscrites dans les énoncés d'un discours argumentatif. Le but est d'identifier l'effet stratégique que la présence de modalités appréciatives peut avoir. Cet effet dépend du rôle argumentatif que joue, dans le débat, l'énoncé dans lequel la modalité apparaît. À partir d'un corpus de 51 textes produits dans le cadre du débat public sur les nanotechnologies organisé en France entre 2009 et 2010, on cherche à illustrer les deux modes différents d'inscription de la modalité appréciative (intégrée et détachée) et à décrire leur effet respectif dans l'énoncé-thèse et dans l'énoncé-argument.

Mots clés: modalité appréciative, adverbes évaluatifs, coups argumentatifs, pragma-dialectique, énoncé-thèse, énoncé-argument

This paper studies the way in which evaluative modality is used in argumentative discourse. The aim is to identify the strategic effect of the various linguistic realisations of evaluative modality in two argumentative moves, namely the move of advancing a standpoint and the move of advancing arguments in support of the standpoint. This effect depends on the distinct function that each of these moves plays in a debate. As a case in point, the strategic effect of evaluative modality is studied in a corpus of 51 texts produced by various stakeholders during the public debate on nanotechnology in France held between 2009 and 2010.

Keywords: evaluative modality, evaluative adverbs, argumentative moves, Pragma-dialectics, standpoint, argument

\section{Introduction}

Cet article vise à montrer comment les choix offerts par le système linguistique contribuent à la détermination du rôle que jouent les énoncés produits par des locuteurs dans une discussion argumentative. Quels sont les moyens linguistiques dont les locuteurs peuvent se servir dans leurs contributions à une discussion argumentative? Quel effet ces moyens linguistiques peuvent-ils avoir sur le discours argumentatif? Répondre à ces questions nécessite de mettre en œuvre une analyse de l'argumentation qui prête attention à la dimension linguistique du discours argumentatif ${ }^{1}$.

1. Voir à cet égard les articles publiés dans le numéro thématique de la revue Verbum coordonné par Marianne Doury (2010). 
La modalité appréciative constitue une ressource linguistique dont disposent les participants à une discussion argumentative. Cette modalité exprime un jugement de valeur ou une réaction affective du locuteur. Elle peut être réalisée dans le discours par une multitude de moyens lexicaux et morphosyntaxiques. Il s'agit, dans cet article, de préciser le rôle que la modalité appréciative peut jouer dans le discours argumentatif, et non pas de décrire cette modalité en tant que telle, ni de décrire le rôle qu'elle joue dans le discours en général. L'approche pragmatique et dialogique de l'analyse du discours argumentatif que j'adopte prend en compte les aspects sémantiques et syntaxiques du langage (voir Plantin, 1996; Van Eemeren et Grootendorst, 2004), sans toutefois prétendre proposer une étude linguistique fouillée du phénomène de l'appréciation. Dans cette perspective, je vais me concentrer sur l'analyse de quelques énoncés argumentatifs tirés d'un corpus de $5 \mathrm{I}$ textes monologaux écrits, produits par des parties prenantes variées lors du débat public sur le développement et les applications des nanotechnologies, qui a eu lieu en France entre 2009 et 2010.

Je présenterai d'abord les différentes marques linguistiques par lesquelles la modalité appréciative peut être réalisée dans le langage. J'examinerai ensuite la pertinence de ces différentes catégories pour une analyse argumentative afin de décrire l'effet de l'emploi des modalités appréciatives dans le discours argumentatif. Les questions qui concernent le développement et les applications des nanotechnologies suscitent, en France comme ailleurs dans le monde, des évaluations de la part des acteurs du débat, qui vont du positif (les nanotechnologies sont qualifiées de «révolution») au négatif (les nanotechnologies sont qualifiées de «menace»). Par ailleurs, les cibles de ces évaluations peuvent être les nanotechnologies et leurs produits ou applications, mais aussi les instances actives derrière ces nanotechnologies (les scientifiques, les industriels, le gouvernement) ainsi que les acteurs qui s'y opposent, et finalement, le débat lui-même. L'étude de l'inscription des modalités appréciatives dans le discours argumentatif produit par les acteurs du débat national sur les nanotechnologies en France, met au jour différentes stratégies employées par les parties prenantes, qui cherchent à renforcer ou à anticiper les évaluations positives ou négatives afin d'affirmer leurs positions respectives.

\section{Modalisation et modalité appréciative}

$4 \quad$ Par modalité appréciative, j'entends l'expression d'une gamme d'attitudes évaluatives à l'égard de l'énoncé. Ces attitudes ne concernent pas le degré de certitude par rapport à ce que le locuteur affirme, mais ont plutôt à voir avec une réaction affective ou un jugement de valeur (voir l'entrée «modalité» dans Charaudeau et Maingueneau, 2002). La modalité appréciative est ainsi distinguée de la modalité épistémique, qui fait référence à l'expression de la certitude du locuteur à l'égard de son énoncé. Ces deux types de modalité relèvent des modalités d'énoncé, qui portent sur l'attitude épistémique ou appréciative de l'énonciateur à l'égard du contenu propositionnel de son énoncé. Les modalités d'énoncé sont à distinguer des modalités d'énonciation, qui concernent le rapport entre l'énonciateur et son interlocuteur ou le rapport 
qu'entretient l'énonciateur avec son propre discours. Ces trois types de modalité (épistémique, appréciative et les modalités d'énonciation) peuvent être considérés comme l'expression de l'inscription du locuteur dans son discours, autrement dit, comme l'expression de la subjectivité (Kerbrat-Orecchioni, 1980)².

Selon Kerbrat-Orecchioni (1980: I20), on peut distinguer deux aspects de la subjectivité: a) l'affectif, qui concerne toute expression d'un sentiment du locuteur; b) l'évaluatif, qui correspond à tout jugement ou à toute évaluation du locuteur. L'appréciation peut être exprimée en termes de bon/mauvais (axiologique, en rapport avec les valeurs), ou passer par une modalisation selon le vrai, le faux ou l'incertain (épistémique, concernant le savoir).

Selon Charaudeau (1992: 599), l'appréciation n'est qu'une sous-catégorie parmi d'autres de la catégorie de modalité qu'il appelle élocutive et qui précise «la manière dont le locuteur révèle sa position vis-à-vis du Propos qu'il énonce». Il remarque que l'appréciation peut porter sur différents domaines de valeur: valeurs morales (je trouve bien/mal que...), valeurs esthétiques (je trouve beau/laid que...), valeurs émotionnelles (je trouve beureux/malbeureux que...), et valeurs utilitaires (je trouve utile/inutile que...). Lorsqu'il recourt à la modalité de l'appréciation définie par Charaudeau, le locuteur prend la responsabilité de l'évaluation qu'il exprime à l'égard du fait présupposé. Par ailleurs, dans l'assertion appréciative du type délocutif, ce rôle du locuteur est neutralisé ${ }^{3}$.

Dans ce qui suit, j'entends par modalité appréciative toute expression d'une évaluation, axiologique ou non axiologique. Si l'on se réfère aux catégories (mentionnées ci-dessous) de la subjectivité distinguées par Kerbrat-Orecchioni (1980) pour dresser une typologie des adjectifs, je retiens donc ce qu'elle recouvre sous le terme d' «évaluatif» (axiologique ou non axiologique), en opposition à l'affectif. Par ailleurs, contrairement à elle, j’exclus le jugement épistémique de l'aspect évaluatif.

\section{L’appréciation : catégorie formelle ou conceptuelle?}

Avant de parler de la réalisation linguistique de la modalité appréciative et de son inscription dans le discours argumentatif en particulier, il est nécessaire de clarifier la différence entre deux approches de la notion d'appréciation, l'une étant plutôt formelle, l'autre, plutôt conceptuelle.

2. Selon Kerbrat-Orecchioni (voir l'entrée «subjectivité» dans Charaudeau et Maingueneau, 2002), c'est à Émile Benveniste que l'on doit le statut véritablement linguistique accordé à la notion de subjectivité. Les notions de modalisation et d'évaluation servent aussi à décrire la présence du locuteur dans son propre discours. En anglais, les termes souvent utilisés, outre evaluation, sont stance et appraisal, ainsi qu'une acception plus large du terme modality. Pour une vue d'ensemble, voir Hunston et Thompson (I999).

3. Cette distinction entre modalité élocutive et modalité délocutive peut être illustrée par la comparaison entre les phrases Je trouve bien que... et Il est bien que..., dont le locuteur peut se servir pour exprimer son appréciation (de manière subjective dans le premier cas et de manière plutôt neutre dans le second). 
Selon une approche formelle, l'appréciation se rattache à des catégories grammaticales et des éléments linguistiques explicites qui expriment un certain jugement de valeur de la part du locuteur à l'égard de son énoncé. Ainsi, on peut identifier des noms affectifs ou évaluatifs, des adjectifs, des verbes et des adverbes ainsi que des éléments comme les temps du verbe, l'intonation ou la ponctuation, qui marquent la subjectivité et l'appréciation dans la langue.

Charaudeau (1992: 573) remarque que l'approche formelle présente certains problèmes liés à la polysémie de marques évaluatives et même, dans certains cas, à leur polyvalence. Prenons les deux exemples suivants, tirés de mon corpus ${ }^{4}$, qui illustrent la polysémie du substantif risque:

[I] En outre, certains nanomatériaux chimiquement «inertes» constituent du fait, notamment de leur caractère non soluble et biopersistant, un risque pour la santé humaine et l'environnement.

(Confédération française des travailleurs chrétiens [CFTC])

[2] Pour la CFTC, il est indispensable de contraindre les industriels à rendre public [sic] les informations dont ils disposent sur les dangers et les risques associés à leurs produits.

(CFTC)

Risque dans l'exemple [I] est synonyme de danger, tandis que dans [2] il est synonyme d'éventualité.

Le problème de polyvalence peut être illustré par l'exemple suivant:

[3] Il y a de nombreux autres métaux, oxydes métalliques, structures carbonées et d'autres matériaux que l'on commence à utiliser à l'échelle nanométrique. Malbeureusement on sait peu de choses à propos de leurs effets néfastes potentiels pour l'homme ou pour l'environnement.

(SEPANSO)

4. Du 15 octobre 2009 au 24 février 20I0, un débat public a été organisé dans I7 villes françaises pour informer le grand public et pour faire entendre les différentes positions sur la question du développement et des applications des nanotechnologies. Un grand nombre de textes ont été produits soit par les organisateurs du débat, soit par les acteurs (citoyens, chercheurs, collectivités, syndicats, associations, etc.), ainsi que par les médias. L'ensemble de ces textes est disponible sur le site officiel du débat: http://cpdp.debatpublic.fr/cpdp-nano/documents/documents-par-type.html. Parmi ces documents figurent des «cahiers d'acteurs», à savoir des brochures de 4 pages rédigées par des personnes morales (groupes parlementaires, collectivités locales, associations, chambres consulaires, syndicats professionnels, organismes publics, parapublics ou privés); voir http://cpdp.debatpublic.fr/cpdp-nano/documents/ liste-cahier-acteurs.html. Dans le texte du compte rendu du débat, les cahiers d'acteurs sont présentés ainsi: «Ces documents normalisés constituent une base de données particulièrement riche puisqu'ils résument les positions d'acteurs très divers, intéressés ou plus ou moins opposés, au développement des nanotechnologies en France». 
Dans l'exemple [3], l'adverbe évaluatif malheureusement, outre sa valeur évaluative, a une valeur oppositive qui le rapproche du connecteur mais ou néanmoins ${ }^{5}$.

Selon une approche conceptuelle, l'appréciation n'est pas nécessairement réalisée dans le discours par des marques linguistiques explicites mais relève d'un processus cognitif continu, qui est actualisé dans le discours par la mobilisation de procédures logico-discursives. Selon Moirand (1995: 86):

[l'évaluation] s'actualise dans des opérations langagières discursives beaucoup moins liées à la langue et liées plutôt à une rhétorique argumentative, à travers des procédures que seule la logique naturelle nous a permis de repérer, grâce aux notions de schématisation, de préconstruit et de représentation.

Kerbrat-Orecchioni (1980: I2I-I3I) propose, elle aussi, un élargissement similaire de la notion de subjectivité, de manière à inclure des processus de constitution du discours comme la sélection et la hiérarchisation de l'information représentée.

Malgré les problèmes de polysémie et de polyvalence liés à l'approche formelle de l'appréciation comme catégorie linguistique plutôt que comme catégorie discursive, je considère que cette approche reste pertinente pour l'objectif de cet article, qui vise à étudier l'effet de l'inscription de l'appréciation dans le discours argumentatif. Si l'on considère l'appréciation en tant que catégorie discursive, on se prive de la possibilité d'étudier son effet dans le discours argumentatif, puisque l'évaluation risque de se confondre avec l'argumentation. Pourtant, toute proposition évaluative ne fonctionne pas, par défaut, comme l'expression d'une thèse dans un discours argumentatif, et l'expression d'une thèse ne se réduit pas nécessairement à l'expression d'une évaluation. Avancer une thèse consiste à prendre position pour ou contre une proposition (dont le contenu peut être descriptif, évaluatif ou injonctif), alors qu'exprimer une évaluation consiste à adopter une attitude positive ou négative à l'égard d'un procès ou de l'objet d'un procès dans une proposition. La nature du jugement, dans le premier cas, est du type «la thèse avancée est tenable/non tenable», tandis que dans le deuxième cas, le jugement peut être de nature épistémique, esthétique, éthique, etc.

C'est pourquoi, dans ce qui suit, je ne parle pas de l'évaluation qui peut être implicitement suggérée dans le discours: une telle approche supposerait une interprétation évaluative du discours qui, pour partie, reviendrait à une analyse et à une interprétation argumentatives. J'assume une approche formelle de l'appréciation, et j'identifie les marqueurs à travers lesquels son inscription dans le discours est rendue explicite.

5. De plus, le fait que certains adverbes comme beureusement ou malbeureusement, ainsi que des adverbes comme étonnamment et paradoxalement, renvoient à une certaine opposition, leur confère aussi un rôle structurant dans le discours, un peu comme des connecteurs pragmatiques tels que par contre et néanmoins (voir aussi Lamiroy et Charolles, 2004). 


\section{Différentes réalisations des modalités appréciatives}

Partant d'une approche formelle de l'appréciation, dans le sens présenté ci-dessus, on peut distinguer les éléments des modalités appréciatives qui font partie du contenu propositionnel de la phrase de ceux qui n'en relèvent pas. Dans le premier cas, on parlera de modalités appréciatives intégrées, tandis que dans le deuxième cas, on parlera de modalités appréciatives détachées.

Guimier (1996: 6), qui étudie les adverbes en -ment en français, propose de distinguer adverbes endophrastiques et adverbes exophrastiques, les uns étant des constituants internes de la phrase qui affectent son contenu, les autres étant des constituants externes de la phrase, qui apportent des informations concernant un ou plusieurs des éléments constitutifs de l'acte d'énonciation. Guimier propose aussi une distinction entre adverbes intra- et extraprédicatifs, distinction de nature syntaxique, tandis que l'opposition endo-/ exophrastique est de nature sémantique. Selon cette distinction, les éléments intraprédicatifs sont intégrés syntaxiquement à la phrase en tant que déterminants du verbe, par exemple: «Pierre sourit bizarrement». En revanche, les éléments extraprédicatifs ne sont pas intégrés à la phrase, comme dans l'exemple: «Bizarrement, Pierre sourit».

La distinction entre modalités intégrées et modalités détachées fait sens lorsqu'on étudie l'effet de leur présence dans des énoncés qui servent à réaliser un «coup argumentatif» dans une discussion, comme avancer une thèse ou des arguments à son appui. Prenons les deux énoncés suivants (empruntés à Guimier, 1996: 106):

Il est heureux que Pierre soit venu.

Heureusement, Pierre est venu.

Dans le premier cas, le locuteur confronté à la réaction «Pourquoi pensez-vous cela?» de l'interlocuteur en désaccord avec lui est censé avancer des arguments pour la thèse «Il est heureux que Pierre soit venu» (le désaccord porte sur la question «Est-il heureux que Pierre soit venu?»). Par contre, le locuteur du deuxième énoncé, confronté à la réaction «Pourquoi pensez-vous cela?», est censé avancer des arguments à l'appui de la proposition «Pierre est venu» (le désaccord porte ici sur la question «Est-ce que Pierre est venu?»). Dans le premier cas, où la modalité appréciative fait partie du contenu propositionnel mis en cause par l'interlocuteur dans une discussion argumentative, je parlerai de modalité appréciative intégrée au contenu propositionnel de l'énoncé (sous 4.I). Dans le second cas, où la modalité appréciative n'est pas questionnée (ou du moins pas directement) par la réaction critique de l'interlocuteur, je parlerai de modalité appréciative détachée (sous 4.2).

\subsection{Modalités appréciatives intégrées}

Il s'agit des éléments linguistiques qui sont des constituants de la phrase ou qui portent sur des constituants de la phrase, comme les noms et les verbes ainsi que 
les adjectifs et les adverbes. Ces éléments participent à la construction du contenu propositionnel de l'énoncé de façon directe.

Ils peuvent être des noms dits affectifs ou évaluatifs (Kerbrat-Orecchioni, 1980; Riegel et al., 2009: 976), c'est-à-dire des noms connotés en termes de niveau de langue (baraque pour désigner un logement misérable, phobie au lieu de crainte, fantasme au lieu de vision), mais aussi des nominalisations que le locuteur peut utiliser pour faire référence aux paroles, actions ou pensées de son interlocuteur. Comme le note Charaudeau (1992), la nominalisation peut jouer un rôle de «reprise anaphorique» en synthétisant la description antérieure d'une ou plusieurs actions tout en rendant l'action plus abstraite. De ce fait, le locuteur peut «importer» son propre jugement sur l'action ou la pensée à laquelle il fait référence, jugement qui s'inscrit dans le choix d'une certaine nominalisation ou d'un substantif évaluatif plutôt que d'un autre.

Autres éléments de la réalisation de la modalité appréciative intégrée par excellence : les adjectifs. On l'a dit, Kerbrat-Orecchioni (1980) fait la distinction entre adjectifs affectifs et adjectifs évaluatifs. Au sein des évaluatifs, elle distingue deux sous-groupes. Les évaluatifs axiologiques impliquent une double norme (interne à la classe de l'objet et relative aux systèmes d'évaluation du locuteur, qui porte par là un jugement de valeur positif ou négatif) : beau/laid, bon/mauvais. Les évaluatifs non axiologiques, quant à eux, impliquent une évaluation qualitative ou quantitative de l'objet indiqué sans énoncer de jugement de valeur ni d'engagement affectif (du moins si l'on s'en tient à leur stricte définition lexicale) : grand, chaud, cher.

Certains verbes, dont le sémantisme exprime un sentiment, une perception subjective, une opinion, un jugement de vérité, peuvent aussi servir à la réalisation de la modalité appréciative dans le discours, en particulier lorsqu'ils sont employés à la première personne. Kerbrat-Orecchioni (1980) distingue les verbes occasionnellement subjectifs (qui n'impliquent un jugement évaluatif que lorsqu'ils sont conjugués à la première personne, comme aimer, apprécier, détester, craindre) et les verbes intrinsèquement subjectifs. Dans ce dernier cas, l'évaluation peut porter soit sur le procès lui-même: criailler, perpétrer, sévir; soit sur au moins un des actants engagés dans le procès dénoté: mériter, risquer, etc.

En ce qui concerne les adverbes, Kerbrat-Orecchioni (1980) inclut dans la catégorie des adverbes subjectifs des adverbes comme vraisemblablement, certainement, vraiment, réellement, ainsi que des adverbes comme franchement, sincèrement, personnellement, ce qui témoigne de l'acception assez large de la subjectivité que l'auteur adopte. Dans l'étude présentée ici, on ne considère comme des adverbes subjectifs que les adverbes de manière, orientés vers le sujet ou le prédicat (ou les deux à la fois), qui dénotent une propriété/qualité du sujet en tant qu'il s'engage dans le procès décrit par le verbe de la phrase. Il s'agit d'adverbes de manière orientés vers le sujet comme anxieusement, tranquillement, brutalement, prudemment, naïvement (voir Guimier, 1996). 


\subsection{Modalités appréciatives détachées}

Par modalités appréciatives détachées, je désigne les éléments linguistiques ou les constructions qui expriment un jugement d'évaluation, et qui sont périphériques aussi bien sémantiquement que syntaxiquement par rapport à la prédication principale. Il s'agit d'abord d'adverbes évaluatifs exophrastiques comme beureusement, malheureusement, curieusement, étonnamment ${ }^{6}$. Comme le note Guimier (I996: III), «les adverbes susceptibles de marquer la modalité appréciative portée sur un contenu propositionnel sont relativement peu nombreux en français», en comparaison avec l'anglais, par exemple. Des groupes prépositionnels comme par chance, par malheur, peuvent aussi être utilisés pour dénoter une appréciation positive ou négative à l'égard de l'ensemble du contenu propositionnel de l'énoncé. Une caractéristique importante de ces adverbes et groupes prépositionnels est qu'ils sont incidents à une phrase complète, et non à un syntagme ${ }^{7}$.

De plus, dans la catégorie des modalités appréciatives détachées, je propose de considérer des structures plus complexes, comme des groupes nominaux, participiaux et des compléments circonstanciels en tête de phrase ou en position incise. Ces «constructions détachées», comme les définit Combettes (1998: 136), ne se trouvent pas dans «des relations de dépendance ou de rection avec d'autres éléments». Selon lui, elles sont caractérisées par leur liberté de position, leur valeur prédicative et l'obligation de coréférence avec un autre constituant ${ }^{8}$. Jackiewicz (20IO) s'appuie sur Combettes pour étudier en particulier les constructions détachées qui dénotent une appréciation. Elle remarque que ces constructions détachées «constituent l'un des lieux privilégiés de l'expression des évaluations» (Jackiewicz, 2010 : I6). Elle cite notamment les exemples qui suivent (Jackiewicz, 2010: 17):

Pragmatique et prudent, Barack Obama s'est bien gardé jusqu'ici de chiffrer son plan de relance dans les infrastructures.

Le conseil des impôts a décidé, très sagement, de supprimer celles dont les effets sont impossibles à évaluer.

Patron respecté, il n'a pas su se faire aimer par ses troupes.

Même s'il s'agit de formes très hétérogènes au niveau syntaxique, ces constructions partagent, au niveau du discours, l'expression d'une évaluation soit à l'égard de l'action

6. À distinguer de l'incidence endophrastique de certains de ces adverbes, que je considère comme une forme de modalité appréciative intégrée, comme le montre l'exemple suivant, emprunté à Guimier (1996: I07): «Le débat s'est heureusement terminé» (= Le débat s'est terminé de manière heureuse).

7. À noter ici que les cas où les adverbes beureusement et malbeureusement sont post-modifiés à l'aide d'un groupe prépositionnel du type pour $N$ ne comptent pas, dans le cadre de cet article, comme des cas de modalité appréciative détachée même s'ils sont syntaxiquement détachés. En effet, la thèse avancée par un tel énoncé dans une discussion argumentative porte sur le commentaire évaluatif, et non sur le contenu de la phrase principale (voir en 4).

8. Ces deux dernières caractéristiques des constructions détachées expliquent que Combettes ne soit pas prêt à considérer des adverbes de manière et des circonstants prépositionnels comme faisant partie des constructions détachées (Combettes, 1998: 30-31). 
ou de l'événement que le verbe désigne (du procès), soit à l'égard des actants, du sujet ou de l'objet de l'action (de l'objet du procès).

Quand on compare les adverbes évaluatifs exophrastiques avec ces constructions détachées, on constate que tous peuvent être analysés comme une sorte de proposition réduite, syntaxiquement détachée du reste de la phrase. Leur usage introduit ainsi un contenu propositionnel qui est présenté au premier plan comme relativement consensuel, sur lequel le locuteur n'attendrait pas, en principe, un désaccord dans une discussion argumentative. Jackiewicz et al. (2009) remarquent que ces constructions détachées «conditionnent de manière sensible l'interprétation du texte, sans faire l'objet d'une assertion explicite». Le commentaire évaluatif est présenté «comme allant de soi ou comme étant communément partagé». C'est en ce sens que je considère que les modalités appréciatives détachées, comparées aux modalités intégrées, jouent un rôle cadratif pour l'énoncé qui sert à avancer un certain "coup argumentatif» dans une discussion.

\section{L'effet de l'inscription des modalités appréciatives dans le discours argumentatif}

Comme on vient de le voir, l'objet de cette étude est la façon dont les modalités appréciatives peuvent être interprétées quand elles sont inscrites dans des énoncés du discours argumentatif. Le but est d'identifier l'effet stratégique que peuvent avoir ces modalités appréciatives en fonction du rôle argumentatif de l'énoncé dans lequel elles apparaissent. Prenons les deux exemples suivants, adaptés d'un texte de mon corpus:

[4] Malbeureusement, la réflexion sur le développement des nanotechnologies ne peut se réduire aux seuls aspects économiques, sanitaires et environnementaux puisque ces nouvelles technologies posent intrinsèquement un grave problème éthique.

[5] La réflexion sur le développement des nanotechnologies ne peut se réduire aux seuls aspects économiques, sanitaires et environnementaux puisque, malbeureusement, ces nouvelles technologies posent intrinsèquement un grave problème éthique.

Dans l'exemple [4], l'adverbe évaluatif malheureusement qualifie la phrase principale qui sert à avancer une thèse dont la justification est exprimée dans la phrase subordonnée, introduite par puisque. Dans [5], l’adverbe évaluatif qualifie la phrase subordonnée qui sert comme argument à l'appui de la thèse avancée dans la phrase principale. Il s'agit de montrer que l'emploi de l'adverbe évaluatif a un effet différent selon le rôle que l'énoncé joue dans une reconstruction argumentative du discours.

Afin de préciser l'effet que l'emploi des modalités appréciatives peut avoir dans le discours argumentatif, il faut d'abord identifier les différents «coups argumentatifs» dans lesquels ces modalités peuvent apparaître ${ }^{9}$. L'approche pragma-dialectique

9. Pour une étude de l'effet stratégique des adverbes illocutifs et épistémiques, voir Tseronis (2013). 
développée par Van Eemeren et Grootendorst (1992, 2004) nous servira de point de départ. Selon cette approche, le discours argumentatif (qu'il soit écrit ou oral) peut être analysé comme un dialogue entre un Proposant et un Opposant à propos de la tenabilité/défendabilité d'une thèse. Le but de ce dialogue est d'arriver à la résolution du conflit d'opinions qui caractérise la situation de communication, situation dans laquelle l'énoncé asserté par le locuteur-Proposant est confronté au doute ou à l'opposition de l'interlocuteur-Opposant. Dans ce dialogue, les deux parties prennent des tours de parole en produisant des coups qui les aident à accomplir des tâches spécifiques à chacune des quatre étapes de la discussion critique, à savoir: l'étape de la confrontation, où le désaccord se manifeste; l'étape de l'ouverture, où les parties prennent en charge les rôles d'Opposant et de Proposant et s'entendent sur les points de départ communs; l'étape de l'argumentation, où le Proposant apporte des arguments et l'Opposant les critique; et l'étape de la conclusion, où les deux parties font le bilan de la tentative de résolution du désaccord.

Parmi les coups argumentatifs que chacune des deux parties peut produire dans une discussion critique, on peut distinguer: (I) l'acte d'avancer une thèse, (2) l'acte de mettre en doute cette thèse et (3) l'acte d'avancer des arguments à l'appui de celle-ci. Le premier et le troisième coups sont accomplis par le Proposant, tandis que le deuxième est accompli par l'Opposant à la thèse.

1. Avancer une thèse: il s'agit d'un acte accompli par le Proposant lors de l'étape de la confrontation. Le Proposant exprime son engagement à l'égard de la tenabilité (thèse positive) ou de la non-tenabilité (thèse négative) du contenu propositionnel de la question qui est problématisée et qui constitue le thème du différend. Trois types de propositions peuvent être mis en doute: des propositions dont le contenu est descriptif ( Les framboises seront rares cette année»), des propositions dont le contenu est évaluatif («Les framboises sont savoureuses cette année»), et des propositions dont le contenu est injonctif ( «Il faut faire pousser des framboises cette année») ${ }^{10}$. Celui qui avance une thèse doit être prêt à la justifier face aux questionnements exprimés ou anticipés de son interlocuteur. Autrement dit, il doit être prêt à assumer la charge de la preuve ${ }^{11}$.

2. Mettre en doute une thèse: il s'agit d'une des réactions possibles de l'interlocuteur (présent ou virtuel) à qui le Proposant s'adresse. L'interlocuteur peut aussi bien être d'accord, partager la même position que le locuteur; mais c'est un cas sur lequel on ne s'attardera pas ici, dans la mesure où il ne débouche pas sur une discussion argumentative. L'interlocuteur peut avoir la position contraire, auquel cas on a une discussion mixte, c'est-à-dire une discussion dans laquelle chacune des deux parties défend une thèse qui

10. Exemples empruntés à Plantin (I996).

11. Sur l'emploi par le Proposant d'adverbes d'énonciation comme clearly, probably, frankly, fortunately pour la gestion stratégique de la charge de la preuve, voir Tseronis (2009). 
s'oppose à la thèse de l'autre; autrement dit, dans une discussion mixte, les deux thèses constituent deux réponses contradictoires à une même question argumentative (voir Van Eemeren et Grootendorst, 1992; Plantin, 1996).

3. Avancer des arguments à l'appui d'une thèse: il s'agit d'un acte accompli par le Proposant au cours de l'étape de l'argumentation. En principe, la charge de la preuve n'incombe pas à celui qui accomplit cet acte, puisque les arguments, contrairement à la thèse, sont censés s'appuyer sur des points de départ communs (des objets d'accord partagés entre le Proposant et l'Opposant).

Afin de mieux décrire l'effet stratégique de l'inscription d'une modalité appréciative dans un coup argumentatif, on peut essayer de répondre à chacune des questions suivantes:

- Est-ce que la modalité appréciative est intégrée au coup argumentatif, ou est-elle juste détachée?

Si elle est intégrée, la modalité devient l'objet même du questionnement, ou joue un rôle essentiel dans la définition et l'identification du coup argumentatif en question. Il s'agit dans ce cas d'une proposition évaluative qui sert à avancer une thèse évaluative ou à avancer un argument. Dans le cas où la modalité appréciative est détachée, son rôle est plutôt cadratif par rapport au coup argumentatif en question. Cela signifie que la modalité appréciative introduit un contenu propositionnel qui ne devient pas directement l'objet de la discussion argumentative, mais qui peut toutefois influencer le développement de l'argumentation de manière indirecte (voir en 4.2$)^{12}$.

- Quelle est la cible de l'évaluation?

La cible de l'évaluation peut être décrite de manière syntaxique ou de manière discursive. On peut, par exemple, identifier la cible à un des constituants essentiels de la phrase (le sujet, le verbe ou le complément) - dans ce cas, il s'agit d'une évaluation endophrastique - ou identifier la cible à la phrase dans son ensemble - évaluation exophrastique. Dans les deux cas, on peut aussi distinguer la cible de l'évaluation en s'attachant aux aspects énonciatifs/argumentatifs. On peut relever au moins trois cibles du commentaire évaluatif: le locuteur lui-même (la personne ou ses propos, à savoir la thèse ou l'argument avancé); l'interlocuteur (la personne ou ses propos, à savoir la thèse ou l'argument avancé); et la situation argumentative dans laquelle le locuteur avance des arguments à l'appui d'une thèse qui est mise en doute par un interlocuteur (présent ou virtuel). Pour ce qui est

12. Jackiewicz (20I0) examine les relations sémantiques (d'opposition, de causalité, d'élaboration) entre le constituant détaché et le noyau prédicatif de la phrase ou entre constituants détachés multiples. Il serait intéressant de voir plus précisément dans quelle mesure ces relations sémantiques interagissent avec des relations argumentatives. 
du débat sur les nanotechnologies, on peut spécifier ces trois cibles comme suit: les nanotechnologies et les produits ou leurs applications; les acteurs engagés dans le développement des nanotechnologies, ou dans l'opposition à ces mêmes nanotechnologies; et le contexte institutionnel dans lequel se déroule le débat ou la situation économique, politique et sociale dans laquelle la question du développement des nanotechnologies se pose.

- Est-ce que le locuteur prend en charge l'évaluation qu'il exprime (discours subjectif) ou pas (discours objectif)?

Comme le notent Perelman et Olbrechts-Tyteca (2008: 243): «Des jugements de valeur, et même des sentiments purement subjectifs, peuvent, par des artifices de présentation, être transformés en jugements de fait». La construction impersonnelle constitue un tel artifice. Les auteurs font la comparaison entre les deux formules suivantes: «Ces pommes ne me disent rien» et «Je n'aime pas ces pommes». Dans le même sens, Charaudeau (I992: 62I) remarque que l'usage des adverbes neutralise la présence du locuteur et de l'interlocuteur dans la représentation de l'énonciation, ce qui n'est pas le cas quand on utilise des verbes personnels ou des tournures personnelles (voir aussi la distinction proposée par Kerbrat-Orecchioni [1980] entre discours objectif et discours subjectif).

\section{Les modalités appréciatives dans les cahiers d'acteurs du débat public sur les nanotechnologies}

Dans cette dernière partie de l'étude, je discute quelques fragments tirés de mon corpus de textes écrits issus du débat public sur le développement et les applications des nanotechnologies en France (voir note 4). À titre d'exemple, j'essaie de préciser l'effet stratégique des modalités appréciatives utilisées dans les coups argumentatifs qui servent à avancer une thèse, ainsi que dans ceux qui servent à avancer des arguments à l'appui d'une thèse.

Les exemples ont été recueillis à l'aide du logiciel Prospéro. Ce logiciel est développé depuis 1995 par Francis Chateauraynaud et Jean-Pierre Chariau et sert pour le traitement semi-automatique de masses de textes qui constituent des dossiers complexes sur des controverses dans l'espace public (Chateauraynaud, 2003). Le logiciel est conçu de façon à faciliter le traitement conjoint du contenu (ce qui est dit), du mode (la façon de dire) et du cadre (le contexte d'énonciation). Ainsi, Prospéro exige de la part de l'utilisateur, l'attribution d'un code à chaque mot du corpus, codage qui génère un vaste réseau conceptuel qui se superpose à l'intégralité des mots du dossier. Des mots ou groupes de mots peuvent être codés selon des catégories variées comme: ENTITÉS (cette catégorie s'applique aux thèmes et aux personnages qui ont pour référents des noms ou des groupes de noms); QUALITÉS (catégorie qui renvoie aux attributs associés aux noms, essentiellement via des adjectifs); EPREUVES (catégorie relative aux types d'action, de transformation ou 
de jugement, qui s'expriment souvent par des verbes); et MARQUEURS (catégorie qui recouvre les modalités, connecteurs, quantificateurs, et qui est portée essentiellement par des adverbes). C'est à l'utilisateur de décider du nombre d'ENTITÉS ou de QUALITÉS, par exemple, qui vont être distinguées, et des listes de mots qui seront codés comme relevant d'une même ENTITÉ ou QUALITÉ. Ainsi, le logiciel offre plusieurs points d'entrée dans le corpus à l'utilisateur, qui peut, dans ses choix, s'appuyer sur les catégories déjà utilisées pour l'analyse d'autres dossiers, ou créer de nouvelles catégories pour un dossier particulier.

Puisque la force argumentative des énoncés n'est pas strictement fonction du sens lexical des mots utilisés ou de la syntaxe qui détermine leur mise en énoncé, les catégories flexibles et les fonctionnalités de requêtes combinatoires offertes par le logiciel Prospéro facilitent la recherche des séquences où une activité argumentative est identifiable. Ceci dit, un retour au texte, par l'analyste lui-même, est toujours nécessaire pour vérifier qu'il s'agit effectivement d'un passage argumentatif. Pour repérer, dans les $5 \mathrm{I}$ textes des cahiers d'acteurs, des passages où une modalité appréciative était inscrite dans un discours argumentatif, j’ai dans un premier temps effectué des requêtes sur l'ENTITÉ «risque» (contenant des mots comme risque et danger) et l'ENTITÉ «bénéfices» (contenant des mots comme bénéfices et avantages), sur des QUALITÉS et des MARQUEURS contenant des adjectifs et des adverbes évaluatifs, ainsi que sur des constructions comme «il est + adj.», «représenter/constituer + adj.», c'est-à-dire sur un ensemble d'éléments dont on considère qu'ils sont susceptibles de réaliser la modalité appréciative dans le discours (voir en 4). Ainsi, j’ai recueilli un certain nombre de fragments que j'ai dû examiner pour ne retenir que ceux qui relevaient d'une activité argumentative. Dans un deuxième temps, j'ai sélectionné parmi ces fragments, ceux dans lesquels la modalité appréciative apparaissait dans un des deux coups argumentatifs spécifiés plus haut. Mon objectif était de chercher des exemples pour illustrer les différentes catégories discutées dans la partie théorique, et non d'identifier des configurations récurrentes ou de quantifier les données recueillies. Je me suis servi du logiciel Prospéro pour assister ma lecture du corpus plus que pour repérer automatiquement tous les passages qui relèvent du phénomène que j'étudie ici.

\subsection{Modalités appréciatives dans l'énoncé-thèse}

Dans l'énoncé qui sert à avancer une thèse, la modalité appréciative peut être intégrée ou détachée. Dans le premier cas, le jugement que la modalité exprime devient le sujet du débat, il s'agit d'une thèse évaluative qui doit être justifiée une fois qu'elle est mise en doute par l'interlocuteur présent ou virtuel. Dans le deuxième cas, la modalité joue un rôle cadratif pour la présentation de la thèse du locuteur; ce cadre lui sert à gérer la charge de la preuve qui lui incombe lorsqu'il avance une thèse (Tseronis, 2009).

\subsubsection{Modalité appréciative intégrée au contenu propositionnel de la thèse}

La modalité appréciative est intégrée à l'énoncé qui sert à avancer une thèse quand il s'agit d'un adjectif employé dans une construction attributive: «X est adj.». Dans ce cas, on a une thèse (positive ou négative) qui est avancée à propos d'une 
proposition évaluative. Le contenu propositionnel de la thèse est un contenu évaluatif. Avancer une thèse revient alors à exprimer une évaluation, comme dans le texte suivant ${ }^{13}$ :

[6] Comme la langue d'Ésope ou plus récemment comme l'énergie nucléaire, les nanotechnologies peuvent être la meilleure ou la pire des choses, selon les précautions que l'on prendra pour les manier. La meilleure des choses, car elles ouvrent des perspectives immenses dans des secteurs d'actualité : > les économies d'énergie: des nanomatériaux peuvent être utilisés pour le transport d'électricité et permettre des gains de l'ordre de $20 \%$. [...] La pire des choses puisque l'incertitude règne sur les effets des nanoparticules sur l'homme et sur l'environnement.

(Association force ouvrière consommateurs $[\mathrm{AFOC}]$ )

La thèse évaluative qui affirme que «les nanotechnologies peuvent être la meilleure ou la pire des choses», est justifiée par les deux énoncés qui suivent. Dans le corpus des 5I cahiers d'acteurs, la cible de l'évaluation portée par les thèses évaluatives des différents acteurs du débat, est surtout les nanotechnologies et leurs produits.

La présence d'un adverbe évaluatif comme heureusement ou malbeureusement peut, sous certaines conditions, faire de la thèse avancée une thèse évaluative, comme c'est le cas dans le fragment suivant:

[7a] Des enjeux essentiels ne sont malbeureusement pas du tout abordés, telles l'atteinte aux libertés individuelles ou la question de la propriété intellectuelle, car avec les nanotechnologies nous allons vers la privatisation du vivant, mais aussi de la matière inerte, voire de la matière hybride.

(Les Amis de la Terre)

Bien que les adverbes évaluatifs soient des éléments détachés syntaxiquement (et sémantiquement) du contenu propositionnel, ils peuvent parfois avoir une incidence endophrastique intraprédicative (voir Guimier, 1996: 107). Dans ce cas, ils sont intégrés au contenu de la thèse avancée et l'argumentation qui suit vient à l'appui du commentaire évaluatif, et non à l'appui de la phrase principale. Dans [7a], l'argumentation avancée à travers la phrase introduite par car vient à l'appui d'une thèse qui peut être formulée ainsi: «Il est malheureux que des enjeux essentiels ne soient pas du tout abordés». Si l'adverbe manquait, l'argumentation n'aurait plus de sens ni de pertinence:

[7b] ?Des enjeux essentiels ne sont pas du tout abordés, telles l'atteinte aux libertés individuelles ou la question de la propriété intellectuelle, car avec les nanotechnologies nous allons vers la privatisation du vivant, mais aussi de la matière inerte, voire de la matière hybride.

13. Dans les fragments cités je mets en italique les mots ou phrases qui servent à la réalisation de la modalité appréciative. Le nom de l'acteur du débat paraît entre parenthèses à la fin de la citation. 
Pour que l'adverbe qui sert à réaliser la modalité appréciative dans l'avancement d'une thèse soit considéré comme intégré et non pas comme détaché, il faut que l'énoncé qualifié par cet adverbe puisse admettre une paraphrase avec une construction impersonnelle comme «il est adj. que...» - où l'adjectif serait issu du même radical que l'adverbe - et que l'argument vienne à l'appui de cette modalité-ci ${ }^{14}$.

La modalité appréciative peut être inscrite dans la thèse en tant que construction impersonnelle : «il (n')est (pas) + adj. de + inf.». Dans ce cas, en fonction de l'adjectif utilisé, la modalité appréciative peut introduire soit une thèse injonctive soit une thèse évaluative. Des adjectifs comme important, indispensable, nécessaire, essentiel, soubaitable, par exemple, renvoient à une thèse injonctive, tandis que des adjectifs comme difficile et regrettable renvoient à une thèse évaluative. Un groupe intéressant est celui des adjectifs légitime, prudent, imprudent: leur usage renvoie à une thèse injonctive dont la mise en œuvre est qualifiée en plus de «légitime», "prudente», etc. Prenons les deux exemples suivants:

[8] Face à l'insuffisance des études et donc face aux incertitudes, il est légitime de s'inquiéter d'un développement important aux conséquences négatives encore mal connues et donc non maîtrisées. Sur ces composés et sur leur présence dans les produits, le consommateur n'a aucune information.

(Familles rurales)

[9] Le rapport de l'AFSSET de juillet 2008 confirmait qu'«au vu des incertitudes quant aux effets sanitaires des nanoparticules, il est prudent de déclarer les nanoparticules comme niveau de danger inconnu et [de] les manipuler avec la même prudence que les matières dangereuses».

(Les Amis de la Terre)

Le choix d'un tel type d'adjectifs aide le Proposant à cadrer sa thèse par rapport à une valeur pragmatique, éthique, au lieu de dire «il faut» ou «nous demandons/voulons que». Notons que l'argumentation qu'on attendrait à l'appui des thèses avancées dans [8] et [9] ne portera pas sur l'usage de l'adjectif prudent ou légitime mais sur l'injonction à l'action qualifiée de «légitime» ou «prudente». L'usage de tels adjectifs, surtout dans une construction impersonnelle où l'évaluation est présentée de manière plus objective (par rapport à une formulation comme "nous trouvons légitime que...», par exemple), aide à objectiver le statut de la thèse avancée, bien qu'elle reste une thèse avancée par un acteur identifiable et susceptible d'être contestée par un autre acteur du débat (voir la distinction entre modalité élocutive et modalité délocutive proposée par Charaudeau [1992], note 3 supra).

14. Sur les deux reconstructions possibles des énoncés qualifiés par des adverbes évaluatifs comme beureusement et malbeureusement en anglais et les implications pour l'interprétation de l'effet stratégique de ce mode de qualification, voir Tseronis (2007). 


\subsubsection{Modalité appréciative détachée du contenu propositionnel de la thèse}

Quand la modalité appréciative est détachée du contenu propositionnel de la thèse, elle joue un rôle de qualificateur, qui encadre cette thèse en ajoutant un commentaire dont on n'attend pas qu'il soit problématisé dans la discussion qui suit. On peut, par exemple, anticiper la réaction de son interlocuteur et compatir avec lui, tout en avançant une thèse dont on s'attend à ce qu'elle ne soit pas partagée:

[Io] Que chacun de nous se rassure, si le passage en revue des technologies utilisées pour générer et mettre en œuvre les nanomatériaux révèle des technologies relativement sophistiquées, celles-ci sont déjà connues depuis plusieurs dizaines d'années déjà $[s i c]$. Ce qui les différencie d'année en année est le lent, patient et formidable travail ingénièrique $[$ sic] de perfectionnement dont elles sont l'objet. Il en est de même des enceintes de plus en plus vastes dans lesquelles ces techniques sont utilisées. Nous comprenons ici l'étonnement de beaucoup qui ont cru «au miracle d'une nouvelle ère»; nous en sommes désolés, ce n'est pas de miracle qu'il s'agit mais bien du résultat des efforts à la fois conjugués (une analyse fine des brevets internationaux le montre) et souvent concurrentiels de milliers de personnes très qualifiées tant aux USA qu'en Europe.

(Fédération interdépartementale de l'environnement avignonnais [FIDEA])

Ici, la thèse selon laquelle «il ne s'agit pas de miracle mais bien du résultat des efforts conjugués et concurrentiels» est cadrée par la phrase incise «nous en sommes désolés». L'auteur du texte manifeste ainsi son anticipation sur le fait que la thèse qu'il avance va à l'encontre des attentes de son public. En «compatissant» (au sens premier de «souffrir avec») avec son public, l'auteur réussit de manière indirecte à renforcer la validité de sa thèse, qui est ainsi présentée comme une thèse qu'il doit avancer même s'il sait qu'elle va déplaire. Dans l'exemple suivant, la construction détachée «regrettable mais bien réelle» qualifie la thèse selon laquelle «[c]ette situation est responsable, pour partie, d'effets dommageables sur notre santé et notre environnement», et a elle aussi pour effet de renforcer la validité de la thèse.

[II] Il va de soit [sic] qu'en la matière, nous sommes nombreux à être les Messieurs Jourdain des nanomatériaux qui, par la force des choses, ne sont pas, et de loin, suffisamment pris en compte par la plupart d'entre-nous [sic], professionnels compris. Cette situation, regrettable mais bien réelle, est ainsi responsable, pour partie, d'effets dommageables sur notre santé et notre environnement (on notera ici, néanmoins, l'évolution rapide des normes «poussières», ces dernières années, dont la dernière en date - la norme sur les $2,5 \mu \mathrm{m}$ - n'est pas encore suffisante et devra continuer d'évoluer pour rencontrer les nanoparticules déjà existantes comme celles qui arrivent).

(FIDEA)

On peut aussi avancer une thèse en la situant par rapport à d'autres positionnements possibles sur le sujet, de façon à mettre l'accent sur le fait qu'il s'agit d'une thèse qui mérite l'attention car elle assume un positionnement médian dans le débat: 
[i2] En d'autres termes, des risques aujourd'hui inconnus seront peut-être décelés demain et à certaines questions légitimes, la science n'a pas toujours les moyens de répondre. Face à cette incertitude fondamentale, seul le rejet systématique de toute innovation technologique garantirait l'absence de nouveau risque, ce qui reviendrait en retour à priver la collectivité de tout bénéfice et perspective de progrès. Entre confiance béate dans le progrès et catastrophisme irrationnel, la CLCV considère que nous n'avons donc d'autre choix que de trouver une voie médiane.

(Consommation, logement et cadre de vie [CLCV])

Dans l'exemple suivant, la thèse selon laquelle «les nanotechnologies restent pour la plupart des citoyens mal connues», est encadrée par une construction détachée, qui fait référence à la divergence des jugements évaluatifs concernant les nanotechnologies, esquissant ainsi le contexte du débat dans lequel cette thèse est avancée :

[13] Annonciatrices de progrès considérables selon les uns, sources de risques imprévisibles pour les autres, les nanotechnologies restent pour la plupart des citoyens mal connues.

(CLCV)

Dans l'exemple suivant, la thèse selon laquelle «les nanotechnologies présentent un potentiel immense de développement et d'application», est introduite par une construction détachée qui fait référence au jugement positif sur les nanotechnologies exprimé par certains:

[I4] Qualifiées de «moteur de la prochaine révolution industrielle», les nanotechnologies présentent un potentiel immense de développement et d'application.

(CFTC)

Dans l'exemple [13], une relation sémantique d'opposition se développe entre la construction détachée et le noyau prédicatif de la phrase, tandis que dans l'exemple [I4], c'est une relation causale (voir Jackiewicz, 2010: 33). Le fait que la modalité appréciative soit détachée dans les deux cas a comme effet que la relation entre la réalité des nanotechnologies affirmée par la thèse et le jugement évaluatif portant sur leur potentiel, est présentée comme connue et partagée par l'auditoire.

Enfin, voici un exemple où les modalités appréciatives, intégrée et détachée, peuvent être combinées:

[I5] Au risque de décevoir beaucoup, la «révolution» nanométrique est le résultat, dans la grande majorité des cas, de la simple croissance du rapport Surface/Volume quand on diminue la taille d'un matériau, d'un objet. Et les lois classiques de la physique comme celle[s] de la chimie qui y sont rattachées sont de fait encore et toujours d'application [...]. Ce qui change ce sont les rendements, souvent améliorés, de ces phénomènes (plus vite, plus lumineux, plus résistant...).

(FIDEA) 

révolution». par les conséquences. avancée y incite: sur l'information.

(CLCV)

Le syntagme prépositionnel sert à réaliser une modalité appréciative détachée du contenu de la phrase principale, et qui concerne le rapport entre le locuteur et l'interlocuteur, tandis que l'usage des guillemets pour évaluer le mot «révolution» compte comme la réalisation d'une modalité appréciative intégrée, comme une autre manière de mettre en doute le statut révolutionnaire des nanotechnologies, au lieu d'affirmer directement que «les nanotechnologies ne constituent pas de

\subsection{Modalités appréciatives dans l'énoncé-argument}

Dans les énoncés qui servent comme arguments à l'appui d'une thèse, la modalité appréciative intégrée finit par constituer une argumentation sur les valeurs ou une argumentation par les conséquences (positives ou négatives). Ainsi, l'argument avancé est un argument qui fait appel à certaines valeurs ou un argument qui évalue comme positif ou négatif un état de fait actuel ou futur, évaluation qui est mobilisée pour soutenir l'action ou l'inaction à laquelle la thèse invite. Dans l'énoncéargument où la modalité appréciative est détachée du contenu propositionnel, le commentaire évaluatif peut être interprété comme une prémisse évaluative qui s'ajoute simplement à l'argument principal afin de soutenir la thèse avancée de manière indirecte, sans que l'argument devienne un argument sur les valeurs ou

\subsubsection{Modalité appréciative intégrée au contenu propositionnel de l'argument}

Les modalités appréciatives sont intégrées à des énoncés qui servent à avancer des arguments pragmatiques ou des arguments sur les valeurs. Van Eemeren et al. (2007: 170), dans leur ouvrage sur les indicateurs argumentatifs, parlent de l'usage de verbes anglais comme destroy, increase, arouse, etc., qui mettent l'accent sur l'aspect négatif du résultat et peuvent servir comme indicateurs d'arguments causaux.

Dans l'exemple suivant, les «peurs irrationnelles» et le «charlatanisme» décrivent un état de fait qui risque de s'installer si l'on ne fait pas d'efforts pour bien informer le public sur les risques et les bénéfices des nanotechnologies, comme la thèse

[16] S'agissant de risques et de bénéfices qui les concernent, les citoyens ont le droit de savoir et de comprendre. Pour cela, un important travail de vulgarisation de l'expertise devrait être mené sous des formes multiples: conférences de consensus, sites internet dédiés, débats publics, émissions de télévision ou de radio... Sans ces efforts de communication, les peurs irrationnelles et le charlatanisme prendront le pas

De manière similaire, dans l'exemple suivant, la possibilité que les débats restent «théoriques» et que les risques restent «incertains» pèse comme argument à l'appui d'une thèse injonctive qui demande l'étiquetage clair des nanoproduits. 
[I7] Pour FNE toute mise sur le marché européen de nanoproduits doit dans les plus brefs délais s'accompagner d'une étiquette détaillée comprenant au minimum: la taille des particules, la concentration en nanoparticules, la structure des particules et du substrat, la nanotechnologie utilisée, la classification (exemple colloïde). Tant qu'il n'y aura pas d'étiquette claire, les débats resteront théoriques, les risques incertains et les responsabilités de chacun ne pourront pas être assumées.

(France, nature, environnement [FNE])

Enfin, voici un cas intéressant où, dans un seul énoncé, on trouve l'argument et la thèse qu'il appuie:

[I8] À la fois sources potentielles de progrès et de risques, les nanotechnologies doivent donc être soumises à une surveillance rapprochée, sous l'égide d'une autorité indépendante et avec le concours de tous les acteurs concernés.

(AFOC)

Le groupe prépositionnel, qui syntaxiquement est détaché du reste de la phrase, qualifie les nanotechnologies de sources potentielles de progrès ainsi que de risques, une appréciation qui compte comme argument à l'appui de la thèse injonctive qui invite à une surveillance rapprochée de ces technologies, thèse introduite par le connecteur donc. Jackiewicz (20I0:34) remarque aussi que la qualité dénotée dans une construction détachée peut être présentée comme la cause de la situation décrite dans la proposition principale.

\subsubsection{Modalité appréciative détachée du contenu propositionnel de l'argument}

Le détachement de la modalité appréciative du contenu propositionnel de l'énoncéargument sert à insérer un commentaire évaluatif qui s'ajoute à l'argument principal avancé à l'appui de la thèse. Puisque la présence d'un commentaire évaluatif sur un contenu propositionnel présuppose ce contenu propositionnel, c'est-à-dire présuppose qu'il est vrai, accepté, etc., on peut imaginer que la modalité appréciative détachée dans l'énoncé-argument finit par renforcer la crédibilité de l'argument principal ${ }^{15}$.

Prenons l'exemple construit, que l'on vient de discuter dans la section 5, où l'adverbe évaluatif malbeureusement qualifie l'énoncé qui sert à avancer un argument à l'appui d'une thèse:

[i9] La réflexion sur le développement des nanotechnologies ne peut se réduire aux seuls aspects économiques, sanitaires et environnementaux puisque, malbeureusement, ces nouvelles technologies posent intrinsèquement un grave problème éthique.

15. Bonami et Godard (2005), qui ont étudié les propriétés sémantiques et pragmatiques des adverbes évaluatifs, finissent par conclure que le commentaire évaluatif est pris en charge par le locuteur mais ne peut pas être mis explicitement en discussion. Il serait utile de mettre cette propriété pragmatique des évaluatifs en perspective dans une étude attentive au discours argumentatif, afin de distinguer l'effet stratégique du commentaire évaluatif juxtaposé à l'énoncé-thèse de l'effet stratégique du commentaire évaluatif juxtaposé à l'énoncé-argument (voir aussi Tseronis, 2009). 
À l'argument qui dit que les nouvelles technologies posent intrinsèquement un grave problème éthique, avancé à l'appui de la thèse selon laquelle «[1] a réflexion sur le développement des nanotechnologies ne peut pas se réduire aux seuls aspects économiques, sanitaires et environnementaux», s'ajoute le commentaire selon lequel le fait que ces nouvelles technologies posent intrinsèquement un grave problème éthique est quelque chose de malheureux, de mauvais. Ce commentaire ne peut pas être seulement compris comme une prémisse supplémentaire à l'argumentation, mais aussi comme une manière indirecte de renforcer la crédibilité de l'argument principal.

Dans le corpus des 5 I cahiers d'acteurs, on n'a pas d'occurrences d'adverbes évaluatifs qualifiant l'énoncé-argument. Pourtant, la modalité appréciative, comme on vient de le voir dans la section 4.2 , peut être réalisée dans le discours par des constituants détachés, qui servent à cadrer l'énoncé qui les suit. C'est le cas dans l'exemple suivant, qui constitue une bonne illustration de la manière dont les modalités appréciatives peuvent être inscrites dans le discours argumentatif:

[20] De plus, l'une des premières mesures réglementaires à être adoptées nous paraît pour le moins aberrante. Il s'agit de celle proposée dans le domaine des cosmétiques pour informer les consommateurs: apposer sur l'étiquette l'indication «nano» à côté du composant concerné. L'intention est louable: laisser le libre choix aux consommateurs et soumettre ces produits, avec leurs atouts et leurs risques, au verdict du marché. Le principal défaut de cette disposition est pourtant évident: cette étiquette n'aura pas de signification pour la très grande majorité des gens. Ou elle aura l'effet inverse d'une mise en garde: dans certains secteurs, le préfixe nano est d'abord synonyme de high-tech et est déjà devenu un argument marketing. Cette étiquette ne rendra pas non plus compte du degré de risque (très variable d'un composant à l'autre).

(Sciences et Démocratie)

La thèse évaluative, qui affirme que «l'une des premières mesures réglementaires à être adoptée est pour le moins aberrante», est justifiée par un argument introduit par la phrase: «Le principal défaut de cette disposition est pourtant évident». Cette phrase, qui affirme que le défaut de la mesure réglementaire dont il est question est évident, sert à renforcer l'argument qui est avancé à l'appui de la thèse évaluative de départ, conformément à l'effet que l'usage d'un adverbe évaluatif comme malbeureusement aurait pu produire dans ce contexte.

\section{Conclusion}

Dans cette étude, je me suis penché sur l'effet stratégique de l'inscription des modalités appréciatives dans le discours argumentatif. Après avoir présenté les différents moyens par lesquels l'expression d'un jugement de valeur ou d'une réaction affective peut être réalisée dans le discours, j’ai étudié de plus près l'inscription de ces modalités dans deux coups argumentatifs, l'énoncé qui sert à avancer une thèse et l'énoncé qui 
sert à avancer des arguments à l'appui d'une thèse. Partant d'une approche plutôt formelle de l'appréciation, selon laquelle celle-ci est rattachée à des éléments ou des catégories linguistiques identifiables, j’ai distingué les modalités appréciatives intégrées au contenu propositionnel de l'énoncé des modalités appréciatives détachées ou juxtaposées à celui-ci. Ainsi, la modalité appréciative intégrée à l'énoncé-thèse finit par faire de la thèse avancée une thèse évaluative, tandis que la modalité intégrée à l'énoncé-argument finit par constituer une argumentation sur les valeurs ou une argumentation par les conséquences (positives ou négatives). En revanche, la modalité appréciative juxtaposée à l'énoncé-thèse ou à l'énoncé-argument joue un rôle cadratif par rapport au contenu propositionnel de ce coup argumentatif et sert à renforcer la crédibilité de la thèse ou de l'argument avancé. Afin de préciser l'effet stratégique d'un choix linguistique dans la réalisation d'un coup argumentatif, il importe non seulement de rendre compte des propriétés sémantiques et pragmatiques des éléments linguistiques en question, mais aussi, et surtout, de prendre en compte le rôle argumentatif spécifique de l'énoncé dans lequel ces éléments figurent, ainsi que le contexte de communication dans lequel les énoncés étudiés fonctionnent comme thèse ou argument dans un débat.

\section{Remerciements}

Cette étude a été menée dans le cadre d'un projet ANR intitulé «Chimères nanobiotechnologiques et post-humanité - Sociologie des controverses sur les mutations du genre humain annoncées par les nanosciences» (responsable scientifique: Francis Chateauraynaud, EHESS). Je voudrais remercier Marianne Doury (laboratoire Communication et politique [LCP], CNRS), responsable de l'axe argumentatif de ce projet, pour la relecture ponctuelle de mon texte et pour son soutien pendant les deux années de mon contrat post-doctoral au LCP. Je tiens aussi à remercier les deux experts anonymes pour la relecture minutieuse et leurs conseils pertinents.

\section{Références}

Bonami, O. et GODARD, D. 2005. Les adverbes évaluatifs dans une approche multidimensionnelle du sens. In I. CHOI-Jonin et al. (éd.), Questions de classification en linguistique. Méthodes et descriptions. Berne: P. Lang: 19-37.

Charaudeau, P. 1992. Grammaire du sens et de l'expression. Paris: Hachette.

Charaudeau, P. et Maingueneau, D. (éd.) 2002. Dictionnaire d'analyse du discours. Paris: Seuil.

Chateauraynaud, F. 2003. Prospéro: une technologie littéraire pour les sciences bumaines. Paris: CNRS Éditions.

Combettes, B. 1998. Les constructions détachées en français. Paris - Gap: Ophrys.

Doury, M. (éd.) 2010. Verbum: L'inscription langagière de l'argumentation 32 (I). Nancy: Presses universitaires de Nancy. 
Guimier, C. 1996. Les adverbes du français: le cas des adverbes en -ment. Paris - Gap: Ophrys. Hunston, S. et Thompson, G. (éd.) 1999. Evaluation in Text: Authorial Stance and the Construction of Discourse. Oxford: Oxford University Press.

Jackiewicz, A. 20Iо. Structures avec constituants détachés et jugements d'évaluation. Document numérique $\mathrm{I}_{3}(3):$ II-40.

JaCKIEwicz, A., Charnois, T. et Ferrari, S. 2009. Jugements d'évaluation et constituants périphériques. In Actes de la $16^{e}$ conférence sur le Traitement automatique des langues naturelles - TALN 2009 (Senlis, 24-26 juin 2009). I-Io. En ligne sur HAL à l'adresse suivante: https://hal.archives-ouvertes.fr/hal-oIoII828/document.

Kerbrat-Orecchioni, C. 1980. L’énonciation. De la subjectivité dans le langage. Paris: A. Colin.

Lamiroy, B. et Charolles, M. 2004. Des adverbes aux connecteurs : simplement, seulement, malheureusement, heureusement. Travaux de linguistique 49 (2): 57-79.

Le Querler, N. 1996. Typologie des modalités. Caen: Presses universitaires de Caen.

MoIrand, S. I995. L'évaluation dans les discours scientifiques et professionnels. Les carnets $d u$ Cediscor 3: 8I-93. En ligne à l'adresse suivante: http://cediscor.revues.org/497.

Perelman, C. et Olbrechts-Tyteca, L. 2008. Traité de l'argumentation. La nouvelle rhétorique [1958]. Bruxelles: Éditions de l'Université de Bruxelles.

Plantin, C. 1996. L'argumentation. Paris: Seuil.

Riegel, M., Pellat, J.-C. et Rioul, R. 2009. Grammaire méthodique du français. Paris: PUF [ $4{ }^{\mathrm{e}}$ éd.].

Tseronis, A. 2007. The Management of the Burden of Proof and Its Implications for the Analysis of Qualified Standpoints: The Case of Evaluative Adverbials. In F. H. VAn Eemeren et al. (éd.), Proceedings of the 6th Conference of the International Society for the Study of Argumentation. Amsterdam: Sic Sat: 1387-1394.

Tseronis, A. 2009. Qualifying Standpoints. Stance Adverbs as a Presentational Device for Managing the Burden of Proof. Utrecht: LOT Publications.

Tseronis, A. 2013. Exploiter la dialogicité argumentative : le cas des adverbes épistémiques et illocutifs qualifiant une thèse. In D. Pirazzini et A. Schiemann (éd.), Dialogizität in der Argumentation : eine multidisziplinäre Betracbtung. Francfort-sur-le-Main: P. Lang: 297-312.

Van Eemeren, F. H. et Grootendorst, R. 1992. Argumentation, Communication, and Fallacies. A Pragma-dialectical perspective. Hillsdale - Londres: L. Erlbaum [traduit en français par S. Bruxelles, M. Doury et V. Traverso, sous la direction de C. Plantin, 1996. La nouvelle dialectique. Paris: Éditions Kimé].

Van Eemeren, F. H. et Grootendorst, R. 2004. A Systematic Theory of Argumentation. The Pragma-dialectical Approach. Cambridge: Cambridge University Press.

Van Eemeren, F. H., Houtlosser, P. et Snoeck Henkemans, A. F. 2007. Argumentative Indicators in Discourse. A Pragma-dialectical Study. Dordrecht: Springer. 